\title{
General Relativistic effects on the conversion of nuclear to two-flavour quark matter in compact stars
}

\author{
Abhijit Bhattacharyya * \\ Department of Physics, University of Calcutta, 92, A. P. C. Road, Kolkata - 700009; INDIA \\ Sanjay K. Ghosh $]^{\dagger}$ Ritam Mallick周 and Sibaji Rahas \\ Centre for Astroparticle Physics 8 Space Science and Department of Physics, \\ Bose Institute, 93/1, A.P.C Road, Kolkata - 700009, INDIA
}

\begin{abstract}
We investigate the General Relativistic (GR) effects on the conversion from nuclear to two-flavour quark matter in compact stars, both static as well as rotating. We find that GR effects lead to qualitative differences in rotating stars, indicating the inadequacy of non-relativistic (NR) or even Special Relativistic (SR) treatments for these cases.

PACS numbers:
\end{abstract}

Strange Quark Matter (SQM), consisting of approximately equal numbers of up $(u)$, down $(d)$ and strange $(s)$ quarks, is the putative true ground state of strong interaction [1], a conjecture supported by model calculations for certain ranges of values for strange quark mass and strong coupling constant [2]. There have been concerted efforts at confirming the existence of Quark-Gluon Plasma (QGP) and SQM, though transiently, in ultra relativistic collisions. On the other hand, QGP and SQM could naturally occur in the cores of compact stars, where central densities of about an order of magnitude higher than the nuclear matter saturation density are expected. Given the very low strangeness fraction in normal nuclear matter (NM), it is almost inevitable that a transition from nuclear (hadronic) to quark matter should proceed through a conversion to an initial stage of (metastable) two flavour quark matter, which should decay to the stable SQM. Thus, neutron stars with sufficiently high central densities ought to get converted to strange, or at least hybrid, stars. These transitions could have observable signatures in the form of a jump in the breaking index and gamma ray bursts $[3,4]$. On the other hand a full quark star may explain the phenomena of observed quasi periodic oscillations [5].

There are several plausible scenarios where neutron stars could convert to quark stars, through a "seed" of external SQM [6], or triggered by the rise in the central density due to a sudden spin-down in older neutron stars [7]. Several authors have studied the conversion of nuclear matter to strange matter under different assumptions [8, 9, 10, 11, 12, 13, 14, 15, 16, 17, 18. They have been summarized in a recent work of ours [19] and for the constraint of space, we do not repeat them here, except to mention that Tokareva et al [15] have lately modelled the hadron to SQM conversion as a single step

\footnotetext{
*Electronic address: abphy@caluniv.ac.in

${ }^{\dagger}$ Electronic address: sanjay@bosemain.boseinst.ac.in

${ }^{\ddagger}$ Electronic address: ritam@bosemain.boseinst.ac.in

$\S$ Electronic address: sibaji@bosemain.boseinst.ac.in
}

process, arguing that the mode of conversion would vary with the temperature as well as the value of bag constant. Berezhiani et al [16], Bombaci et al 17] and Drago et al [18] suggested that the formation of SQM may be delayed, if the deconfinement process takes place through a first order transition [20] so that the purely hadronic star can spend some time as a metastable object.

In our recent work [19], we have argued that the conversion process is a two step process. The first process involves the deconfinement of nuclear to two-flavour quark matter and the conversion process takes some milliseconds to occur. The second process deals with the conversion of excess down quarks to strange quarks, which occurs via weak interaction, forming a stable SQM and the time taken for this process to occur is of the order of 100 seconds. This work was restricted to the case of a static neutron star and only a SR treatment. To the best of our knowledge, GR effects in such processes have not yet been addressed in the literature. In this letter, we consider GR effects in both static and rotating stars and find qualitatively new results.

As in [19], we use the Nonlinear Walecka model for the nuclear matter euqation of state (EOS). For the sake of brevity, we do not repeat the details here. Suffice it to say, the star is assumed to be composed of only nucleons. The metric describing the structure of the star, is given by [21]

$$
\begin{array}{r}
d s^{2}=-e^{\gamma+\rho} d t^{2}+e^{2 \alpha}\left(d r^{2}+r^{2} d \theta^{2}\right)+ \\
e^{\gamma-\rho} r^{2} \sin ^{2} \theta(d \phi-\omega d t)^{2}
\end{array}
$$

The four gravitational potentials $\alpha, \gamma, \rho$ and $\omega$ are functions of $\theta$ and $r$ only. Once these potentials are known, one can calculate the observed properties of the star. We solve the Einstein's equations for the three potentials $\gamma, \rho$ and $\omega$, using Green's function technique [3, 22] and determine the fourth potential $\alpha$ from the other three potentials. The solution of the star is obtained from the 'rns' code [23], which requires the EOS and a central density as inputs and returns various gravitational potentials and hydrodynamic parameters as outputs. We solve this code for a static as well as for rotating stars with different ve- 
locities, upto the mass-shedding limit or the Keplerian velocity. The results are, of course, as expected; rotation induces a change in the star to an oblate-spheroid shape.

To study the GR effects on the conversion of nuclear to two flavour quark matter, we heuristically assume the existence of a combustive phase transition front of infinitesimal thickness, and study the outward propagation of the front, using GR hydrodynamical equations of motion. First we consider a non-rotating neutron star, where the geometry of the problem is one-dimensional and then extend our calculation to a rotating neutron star, where an extra dimension is needed. Let us assume that the conversion front is generated at the center of the star, and it propagates outwards through the star with a certain velocity, dictated by the initial conditions and hydrodynamical equations, converting the nuclear matter to two-flavour quark matter. Employing the conservation conditions 24, 25] and further employing the entropy condition [26], we determine the flow velocity of matter in the two phases, given by

$$
v_{1}^{2}=\frac{\left(p_{2}-p_{1}\right)\left(\epsilon_{2}+p_{1}\right)}{\left(\epsilon_{2}-\epsilon_{1}\right)\left(\epsilon_{1}+p_{2}\right)},
$$

and

$$
v_{2}^{2}=\frac{\left(p_{2}-p_{1}\right)\left(\epsilon_{1}+p_{2}\right)}{\left(\epsilon_{2}-\epsilon_{1}\right)\left(\epsilon_{2}+p_{1}\right)} .
$$

It is possible to classify the various conversion mechanisms by comparing the velocities of the respective phases with the corresponding velocities of sound, denoted by $c_{s i}$, in these phases. For the conversion to be physically possible, velocities should satisfy an additional condition, namely, $0 \leq v_{i}^{2} \leq 1$; here we have used natural units $\hbar=c=K=1$. If we plot different velocities against baryon number, we get curves from which we could calculate the initial velocity of the front at the centre of the star [19]. Starting with this initial velocity, we investigate the evolution of the front with time under GR effect; to this end, we first have to derive the appropriate continuity and Euler's equations for the metric [eq. (1)]. Treating both nuclear and strange matters as ideal fluids, the system is governed, together with the metric and the EOS, by the Einstein's equation $R_{i}{ }^{k}-\frac{1}{2} \delta_{i}{ }^{k} R=\kappa T_{i}{ }^{k}$ and the equation of motion $T^{k}{ }_{i ; k}=0$ [27].

For adiabatic motion, we have,

$$
\begin{array}{r}
\frac{d\left(\varpi u_{i}\right)}{d s}+\frac{\partial \varpi}{\partial x^{i}}=\frac{\varpi}{2} u^{k} u^{l} \frac{\partial g^{k l}}{\partial x^{i}}+T \frac{\partial \sigma}{\partial x^{i}} \\
\frac{\partial}{\partial x^{k}}\left(\frac{u^{k}}{V} \sqrt{-g}\right)=0 ; \quad \frac{\partial \sigma}{\partial s}=0
\end{array}
$$

where $u^{i}$ signifies the four velocity, $\varpi$ and $\sigma$ are the enthalpy and entropy, respectively. For an isentropic process, i.e. $\sigma=$ constant, the term $T \frac{\partial \sigma}{\partial x^{i}}$ goes to zero.

The above two equations are the starting point for deriving the appropriate continuity and Euler's equations. If we want the equation for $\frac{\partial v}{\partial r}$, we only need to consider the variation of the gravitational potentials with $r$ and the terms containing partial derivatives w.r.t $\theta$ can be neglected. For our metric, $\sqrt{-g}$ comes out to be $\sqrt{-g}=e^{\gamma+2 \alpha} r^{2} \sin \theta$; the four vectors are also calculated from the metric. To derive the hydrodynamical equations, we use 'auxiliary' time $d \tau$ instead of ordinary time $d t$, defined as $d \tau=\frac{\sqrt{g_{r r}}}{\sqrt{g_{t t}}} d t$.

Calculating these values from the metric and putting them in eqn. (4) and (5), we get the continuity and the Euler's equations, given by

$$
\begin{array}{r}
\frac{1}{\varpi}\left(\frac{\partial \epsilon}{\partial \tau}+v \frac{\partial \epsilon}{\partial r}\right)+\frac{1}{W^{2}}\left(\frac{\partial v}{\partial r}+v \frac{\partial v}{\partial \tau}\right)+\frac{2 v}{r}+\frac{v}{r} \cot \theta \\
=-v\left(\frac{\partial \gamma}{\partial r}+\frac{\partial \alpha}{\partial r}\right) \\
\frac{1}{\varpi}\left(\frac{\partial p}{\partial r}+v \frac{\partial p}{\partial \tau}\right)+\frac{1}{W^{2}}\left(\frac{\partial v}{\partial \tau}+v \frac{\partial v}{\partial r}\right) \\
=\frac{1}{2}\left(A \frac{\partial \gamma}{\partial r}+B \frac{\partial \rho}{\partial r}+C \frac{\partial \omega}{\partial r}+E\right),
\end{array}
$$

where, $v$ is the r.m.s velocity and $W$ is the inverse of the Lorentz factor. This system of equations differs from its SR counterpart in the appearance of the gravitational potentials, describing the gravitational forces acting on the fluid in its own gravity field. Once the two basic equation are set, we now proceed exactly as in [19]. We define $v$ as the front velocity in the nuclear matter rest frame and $n=\frac{\partial p}{\partial \epsilon}$ is the square of the effective sound speed in the medium. $d \tau$ and $d r$ are connected by the relation

$$
\frac{d r}{d \tau}=v G
$$

where $G$ is given by

$$
G=\sqrt{\frac{e^{\gamma+\rho}-e^{\gamma-\rho} r^{2} \omega^{2} \sin ^{2} \theta}{e^{2 \alpha}}}
$$

The other parameters of the equation are defined as

$$
\begin{gathered}
A=\frac{v \omega r \sin \theta}{C 1}-1 ; \quad E=\frac{2 \omega^{2} r \sin \theta}{C 1}+\frac{2 \omega^{2} e^{\gamma-\rho} r \sin \theta}{A 1} ; \\
B=\frac{B 1}{A 1}-\frac{v \omega r \sin \theta}{C 1} ; \quad C=\frac{2 \omega e^{\gamma-\rho} r^{2} \sin \theta}{A 1}+\frac{v r \sin \theta}{C 1}
\end{gathered}
$$

where

$$
\begin{array}{r}
A 1=e^{\gamma+\rho}-e^{\gamma-\rho} r^{2} \omega^{2} \sin ^{2} \theta ; \\
B 1=-e^{\gamma+\rho}-e^{\gamma-\rho} r^{2} \omega^{2} \sin ^{2} \theta ; \\
C 1=\sqrt{r^{2} \omega^{2} \sin ^{2} \theta-e^{2 \rho}} ;
\end{array}
$$

After a bit of algebra, we get a single differential equation for $v$ :

$$
\frac{\partial v}{\partial r}=\frac{W^{2} v[K+K 1]}{2\left[v^{2}(1+G)^{2}-n\left(1+v^{2} G\right)^{2}\right]} .
$$

where,

$$
\begin{aligned}
& K=2 n\left(1+v^{2} G\right)\left(\frac{\partial \gamma}{\partial r}+\frac{\partial \alpha}{\partial r}+\frac{2}{r}+\frac{\cot \theta}{r}\right) \\
& K 1=(1+G)\left(A \frac{\partial \gamma}{\partial r}+B \frac{\partial \rho}{\partial r}+C \frac{\partial \omega}{\partial r}+E\right)
\end{aligned}
$$


$\omega=0$ and $\sin \theta=1$ in this equation yield the equation for the static star and if we put all potentials equal to zero, we recover our equation for the SR case [19].

Integrating eq. (10) over $r$ from the center to the surface, we obtain the propagation velocity of the front along the radial direction. The initial condition, i.e, the velocity at the center of the star, should be zero from symmetry considerations. On the other hand, the $1 / r$ dependence of the $d v / d r$ in eqn. (10) suggests a steep rise in velocity near the center of the star.

Our calculation proceeds as follows. Having constructed the density profile of the star for a fixed central density, eqns. (2) and (3) specify the respective flow velocities $v_{1}$ and $v_{2}$ of the nuclear and quark matter in the rest frame of the front, at a radius infinitesimally close to the center of the star. This would give us the initial velocity of the front $\left(-v_{1}\right)$, at that radius, in the nuclear matter rest frame. With this, we integrate eqn. (10) outwards along the radius of the star. The solution gives the variation of the velocity with position as a function of the time of arrival of the front. Using this velocity profile, we can calculate the time required to convert the whole star using the relation $\frac{d r}{d \tau}=v G$.

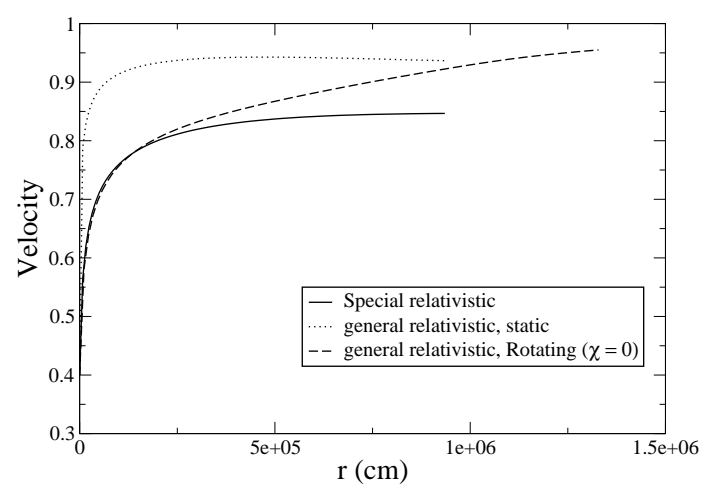

FIG. 1: Variation of velocity of the front along the radial direction of the star for three different cases, namely SR, nonrotating GR and rotating GR.

For a static star, being spherically symmetric, the problem is rather simple; for a rotating star, however, the asymmetry has to be taken care of. For that, we would have to introduce a new parameter $\chi=\cos \theta$, along the vertical axis of the star. We start our calculation by choosing the central density of the star to be 7 times the nuclear matter density, for which the Keplerian velocity of the star is $0.67 \times 10^{-4} \sec ^{-1}$ (the rotational velocities given in fig. (2) are all in units of $10^{-4} \mathrm{sec}^{-1}$ ). For this central density, the initial velocity of the front comes out to be 0.45 . For a complete understanding of the GR effect, comparison with respect to SR is desirable. After solving the differential equation for static and rotating star, we have plotted in fig. (1) the propagation velocity of the front along the radial direction of the star for three cases. The unbroken curve is for the SR case, the bro-

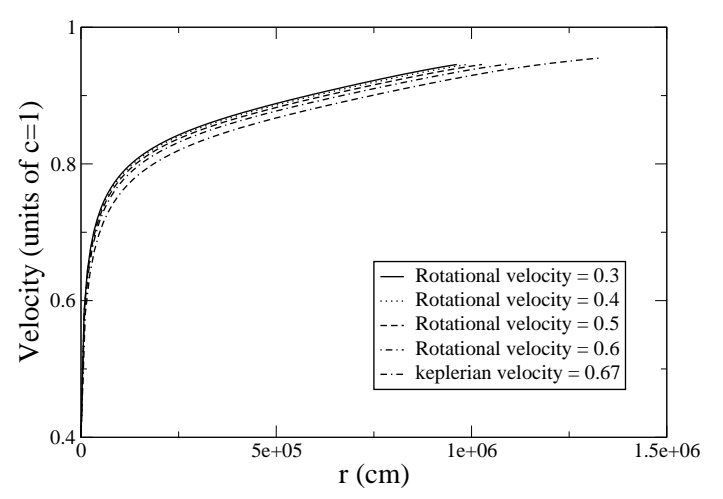

FIG. 2: Variation of velocity of the front along the radial direction for different rotational velocity of the star.

ken curve for non-rotating GR case and the dotted curve for the rotating GR case with $\chi=0$, i.e at the equator. As expected, for all the three cases, the velocity shoots up near the center of the star and saturates at a certain velocity for large radii. This is due to the asymptotic behaviour of the differential equations. It can also be clearly seen that the GR effect increases the velocity of the front considerably (maximum by $30 \%$ ) and the effect is most pronounced for the static case. The rotational effect of the star seems to suppress the GR effect and therefore the velocity of the front decreases. The result becomes clearer if we look at fig. (2) where we have plotted the front velocity with equatorial radius for different rotational velocities; as the rotational velocity increases, the velocity of the front decreases.

From fig. (3), we find that the front velocity is maximum along the polar direction and minimum along the equator. This is quite an interesting result as the equator has the maximum length and the front takes the maximum time to convert nuclear matter to two-flavour quark matter in that direction than towards the pole. Therefore, at any particular instant of time, we may have a situation where the polar part of the star has been con-

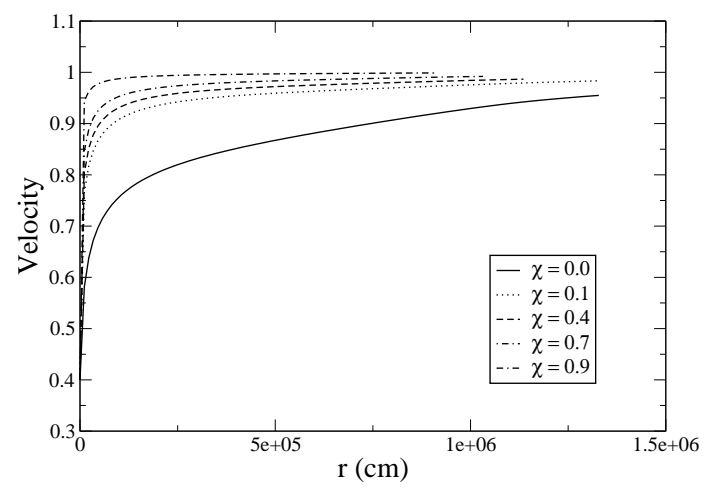

FIG. 3: Variation of velocity of the front along the radial direction for different $\chi$. 


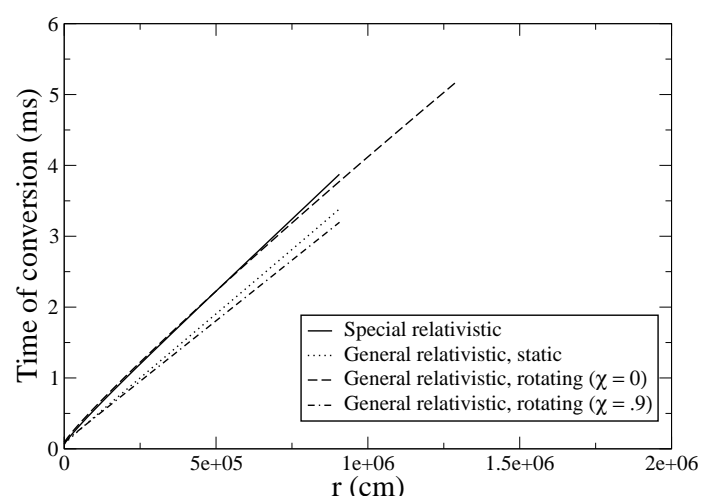

FIG. 4: Variation of time of arrival of the front at certain radial distance for different cases.

verted while along the equatorial direction, the front is still propagating. The result is interesting but not unexpected as at the pole the EOS is much steeper than at the equator (and we have used the slope of the EOS, ' $n$ ' to calculate the front velocity). At some distance from the center of the star, the propagation front breaks up into several distinct fronts propagating with different velocities in different directions.

From fig. (4) we can see that the time taken by the conversion front to convert the neutron star to two-flavour quark star is of the order of few $m s$. The static star takes the minimum time $(3.3 \mathrm{~ms})$ whereas the rotating star takes the maximum time $(5.1 \mathrm{~ms})$ due to the enlarged equatorial radius. The polar part of the star needs much lesser time for conversion $(3.1 \mathrm{~ms})$, even less than static star, as its radius gets compressed.

To summarize, we have shown in this letter that the conversion of nuclear matter to quark matter in compact stars, especially rotating stars which are more realistic than static stars, is strongly affected by GR effects. The emergence of different conversion fronts, propagating with different velocities along different radial directions, is a novel finding, not anticipated by Newtonian or SR calculations. It remains to be explored whether the incorporation of dissipative effects materially changes the conclusion. Intuition would point to a dampening of motion under dissipation, but the relative gradients between the different fronts may also be accentuated, with important physical consequences. Involved though it is, such an investigation is on our immediate agenda.

\section{Acknowledgments}

R.M. thanks CSIR for financial support. A.B. thanks CSIR for support through the project 03(1074)/06/EMR-II. S.K.G. and S.R, in particular, thank DST for support under the IRHPA scheme.
[1] E. Witten, Phys. Rev. D30, 272 (1984)

[2] E. Farhi and R. L. Jaffe, Phys. Rev. D30, 2379 (1984)

[3] A. Bhattacharyya, S. K. Ghosh, M. Hanauske and S. Raha, Phys. Rev. C71, 048801 (2005)

[4] A. Bhattacharyya, S. K. Ghosh and S. Raha, Phys. Lett. B635, 195 (2006)

[5] A. Bhattacharyya and S. K. Ghosh, Mod. Phys. Lett. A22, 1019 (2007)

[6] C. Alcock, E. Farhi and A. Olinto, Astrophys. J. 310, 261 (1986)

[7] N. K. Glendenning, Nucl. Phys. (Proc. Suppl.) B24, 110 (1991); Phys. Rev. D46, 1274 (1992)

[8] A. Olinto, Phys. Lett. B192, 71 (1987); Nucl. Phys. (Proc. Suppl.) B24, 103 (1991)

[9] M. L.Olesen and J. Madsen, Nucl. Phys. (Proc. Suppl.) B24, 170 (1991)

[10] H. Heiselberg, G. Baym and C. J. Pethick, Nucl. Phys. (Proc. Suppl.) B24, 144 (1991)

[11] G. Lugones, O. G. Benvenuto and H. Vucetich, Phys. Rev. D50, 6100 (1994)

[12] O. G. Benvenuto, and J. E. Horvarth, Phys. Lett. B213, $516(1988)$

[13] O. G. Benvenuto, J. E. Horvarth and H. Vucetich, Int. J. Mod. Phys. A4, 257 (1989); O. G. Benvenuto and J. E. Horvarth, Phys. Rev. Lett. 63, 716 (1989)

[14] H. T. Cho, K. W. Ng and A. W. Speliotopoulos, Phys. Lett. B326, 111 (1994)

[15] I. Tokareva, A. Nusser, V. Gurovich and V. Folomeev,
Int. J. Mod. Phys. D14, 33 (2005)

[16] Z. Berezhiani, I. Bombaci, A. Drago, F. Frontera and A. Lavagno, Astrophys. J. 586, 1250 (2003)

[17] I. Bombaci, I. Parenti and I. Vidana, Astrophys. J. 614, 314 (2004)

[18] A. Drago, A. Lavagno and G. Pagliara, Phys. Rev. D69, 057505 (2004)

[19] A. Bhattacharyya, S. K. Ghosh, P. Joarder, R. Mallick and S. Raha, Phys. Rev. C74, 065804 (2006)

[20] J. Alam, S. Raha and B. Sinha, Phys. Rep. 273, 243 (1996)

[21] G.B. Cook, S.L. Shapiro and S.A. Teukolsky, Astrophys. J. 422, 227 (1994)

[22] H. Komatsu, Y. Eriguchi and I. Hachisu, Mon. Not. R. Astron. Soc. 237, 355 (1989)

[23] N. Stergioulas and J. L. Friedman, Astrophys. J. 444, 306 (1995)

[24] A. M. Gleeson and S. Raha, Phys. Rev. C26, 1521 (1982)

[25] A. M. Anile, Relativistic fluids and Magneto-fluids : with application in Astrophysics and Plasma Physics, Cambridge University Press, U.K. (1989)

[26] M. Gyulassy, K. Kajantie, H. Kurki-Suonio and L. McLerran, Nucl. Phys. B237, 477 (1984)

[27] Y. Raizer and Y. Zeldovich, Physics of shock waves and high temperature hydrodynamic phenomena, Academic Press, New York (1967) 